\section{$\underset{\substack{\text { hommes } \\ \text { \& migrations }}}{ }$}

\section{Hommes \& migrations}

Revue française de référence sur les dynamiques

migratoires

$1306 \mid 2014$

Ecriture et migration

\title{
Écriture et migration
}

Pérégrinations avec des adolescents scolarisés tardivement

Joanna Lorilleux

\section{(2) OpenEdition}

1 Journals

\section{Édition électronique}

URL : http://journals.openedition.org/hommesmigrations/2790

DOI : 10.4000/hommesmigrations.2790

ISSN : 2262-3353

Éditeur

Musée national de l'histoire de l'immigration

\section{Édition imprimée}

Date de publication : 1 avril 2014

Pagination : 11-20

ISBN : 978-2-919040-27-8

ISSN : 1142-852X

Référence électronique

Joanna Lorilleux, «Écriture et migration », Hommes \& migrations [En ligne], 1306 | 2014, mis en ligne le 01 avril 2017, consulté le 02 mai 2019. URL : http://journals.openedition.org/hommesmigrations/2790 ; DOI : 10.4000/hommesmigrations.2790 


\section{ÉCRITURE ET MIGRATION PÉRÉGRINATIONS AVEC DES ADOLESCENTS SCOLARISÉS TARDIVEMENT}

par JOANNA LORILLEUX, doctorante en sociolinguistique et didactique des langues, université François Rabelais-Tours (EA 4246 PREFics - DYNADIV).

L'apprentissage du français par des élèves dont ce n'est pas la langue maternelle demeure une gageure pour l'institution scolaire, particulièrement l'écrit. L'intégration de jeunes migrants, peu ou pas scolarisés avant leur arrivée, pose la question de leurs représentations de l'écrit. La mise en place d'ateliers d'arts plastiques centrés sur ces représentations, sollicitant les références linguistiques et culturelles plurielles des élèves, favoriserait l'appropriation de la langue de l'école.

Ce texte repose sur une recherche en didactique des langues visant à comprendre l'évolution des rapports à l'écrit de jeunes migrants allophones (roms, notamment) ${ }^{1}$, à l'occasion d'une première scolarisation tardive. La coconstruction des représentations de l'"écrit" avec des collégiens réputés faiblement littératiés ${ }^{2}$ est l'objectif qui a présidé à l'élaboration d'un projet d'ateliers d'arts plastiques en classe. Créations plastiques, écrits, récits, entretiens et débats mamènent aujourd'hui à reconsidérer les éléments qui me semblaient fondamentaux à l'époque et à envisager autrement le lien qui peut être fait entre écriture et migration chez les jeunes avec lesquels j'ai travaillé. Avant de présenter les éléments d'orientation sous-jacents à la mise en œuvre d'ateliers d'arts plastiques, je peindrai donc le paysage théorique et empirique dans lequel est né mon questionnement. Je rendrai compte également des éléments moteurs, notionnels et expérientiels qui m'incitent aujourd'hui à déplacer la problématique de l'accès au savoir vers celle de la (trans)formation.

\section{Écrit et intégration scolaire}

L'ethnicisation d'une catégorie d'élèves constitue une transgression des lois fondamentales de l'école française. Elle permet pourtant de jeter un regard particulier sur des relations sociales scolaires pos- 
siblement spécifiques, au prisme des relations interethniques. Pour Max Weber, "les groupes ethniques ne supposent [ni une réelle communauté d'origine, ni] une réelle activité communautaire. Ils n'existent que par la croyance subjective qu'ont leurs membres de former une communautée". À quoi s'ajoute l'autre revers de la frontière entre un "nous" et un "eux" construits socialement, celui de l'attribution d'identités. Des individus et des groupes se "disent" constitués, d'autres se pensent extérieurs à eux, et ces appartenances engendrent ou impliquent des représentations qui influent sur les relations sociales entre ces groupes et entre les individus qui les composent. Ces influences sont d'autant plus notables quand la dichotomie eux/nous s'exerce dans des relations entre un groupe dit "minoritaire" et un groupe dit "majoritaire". Une situation particulière m'est apparue, que j’avais formulée comme suit : "De nombreux élèves roms migrants n'entrent pas dans les apprentissages du lire-écrire." Les groupes impliqués dans cette relation sociale sont composés d'une part de l'institution scolaire et de ses représentants, et d'autre part d'élèves s'identifiant et/ou identifiés comme "roms". L'analyse croisée des textes institutionnels relatifs aux structures accueillant ces enfants et d'entretiens menés auprès des élèves concernés, de leur famille et de leurs enseignants, montre qu'il n'y a pas de réelle congruence entre les 
attentes des uns et des autres quant à la fréquentation de l'école.

Les enseignants ne s'expliquent pas ce qu'ils qualifient d'absence d'apprentissage chez les élèves, et qu'ils vivent comme un échec. Les familles, elles, se disent satisfaites de la scolarisation des enfants et ne semblent pas percevoir négativement la situation. Les entretiens menés montrent que l'attente première des familles face à lécole concerne principalement la normalisation de leur situation (administrative, notamment) : la scolarisation doit permettre aux enfants de mener leur vie en France. À l'inverse, les enseignants et les textes visent des apprentissages de savoirs et savoir-faire, qui doivent mener à l'acquisition de l'autonomie. Il semble donc y avoir là un premier malentendu (ou mal-attendu réciproque?).

Selon le point de vue adopté, la relation dans laquelle sont engagés familles, élèves et enseignants joue un rôle potentiel d'inclusion (voie d'entrée dans la société française) ou, au contraire, d'exclusion (élève hors norme, différent, en échec).

\section{Institution et centralité de l'écrit: le rapport épistémique au savoir}

La mobilisation de catégories ethniques (fondées sur la croyance subjective d'appartenance à un groupe) permet d'envisager le rôle de facteurs culturels qui distingueraient des groupes entre eux, notamment sur la question du rapport à l'écrit. Pour ce qui est de l'institution scolaire, Bernard Charlot montre l'essence épistémique du rapport au savoir construit et proposé (imposé) par l'école française. Pour lui, le savoir n'existe que pour "le sujet engagé dans un rapport au savoir". Il s'agit d'un "sujet sachant qu'il sait". Cette relation du sujet connaissant à son monde résulte du rapport d'objectivation-dénomination ${ }^{6}$ - donc d'une relation au langage. Le savoir y est un objet qui s'acquiert, un contenu intellectuel qui peut être transmis. Il est à distinguer de l'apprentissage de savoir-faire ou savoir-être. Cet objet est ontologiquement défini par le caractère scriptural de ses sources et de son évaluation, mais aussi des outils qui permettent d'y accéder?. Ce savoir-objet - que l'institution scolaire française a construit et pose comme allant de soi - prend forme à travers le langage, notamment écrit, qui le distancie apparemment du sujet. Ce processus constitue à la fois le savoir-objet et le "sujet-sachant-qu'il-sait."

Dans cette perspective, le caractère scriptural du savoir constitué comme tel par l'école est central : outre le fait que la mise à lécrit doit permettre de distancier ce savoir du sujet apprenant, c'est l'écriture même qui permet de poser le langage comme objet de savoir. L'écriture doit constituer un moyen d'apprendre tout autant quelle doit permettre à l'apprenant de restituer ses savoirs, afin que l'enseignant puisse en juger.
Les enseignants ne s'expliquent pas ce qu'ils qualifient d'absence d'apprentissage chez les élèves, et qu'ils vivent comme un échec. Les familles, elles, se disent satisfaites de la scolarisation des enfants et ne semblent pas percevoir négativement la situation.
La centralité de l'écrit dans

la définition d'un rapport épistémique au savoir et l'enjeu de l'investissement du sujet amènent à s'interroger sur l'engagement d'élèves dits "en échec dans l'apprentissage du lire-écrire" dans ce rapport au savoir particulier.

Les élèves avec lesquels j’ai travaillé n'ont pas (ou peu) été scolarisés avant leur arrivée en France. J'avais donc supposé que la migration, qui les a mis en contact avec l'école (française), les avait confrontés pour la première fois à un type de rapport au savoir tel que décrit plus haut. 
La migration générée par - et générant - une "conjonction de circonstances ${ }^{8 "}$ aurait fait varier la place de l'écrit et la situation des élèves sur un continuum de littératie ${ }^{9}$ relatif au contexte de son exercice, le rôle de l'école étant dès lors d'accompagner l'élève dans ce déplacement épistémologique. Une des modalités possible de cet accompagnement consiste en l'explicita-

L'école devrait donc, tion des codes scolaires. C'est pour n'être pas coupée de ses élèves, ne pas les amputer de leurs langues et de leur rapport

à elles. Ne pas les ignorer, mais au contraire les accueillir et s'appuyer sur elles, qu'elles soient des variétés du français ou des langues plus éloignées. l'hypothèse sous-jacente à la notion d'empowerment" développée en France par François Dubet ${ }^{10}$. Pourtant, la rencontre d'adolescents migrants scolarisés tardivement laisse entrevoir des pratiques d'écriture antérieures et contemporaines à leur venue en France que l'on pourrait qualifier d'expertes, en dépit desquelles ces jeunes sont perçus par les représentants de l'institution scolaire comme faiblement littératiés. L'action menée à l'occasion de cette recherche repose sur la mise en tension des notions suivantes : migration, intégration scolaire et acculturation à une forme scolaire de littératie, travaillées au prisme de l'hétérogénéité, celle de l'écriture et celle des élèves qui composent les classes et plus largement l'école.

\section{La pensée écran ou l'origine picturale de l'écriture}

Les approches du rapport au savoir et à l'écrit semblent fondées sur le postulat d'une essence verbale de l'écrit. Anne-Marie Christin insiste sur le métissage qui donne naissance à l'écriture : celui du verbal et du graphique, à travers le support, qui devient essentiel à la naissance de l'écrit.

Cette importance accordée au support, à l'écran - qui permet non seulement de représenter les phénomènes, de les figurer, mais qui les symbolise -, le pose comme premier, fondamental à l'apparition de l'écriture. C'est le fait d'inscrire une représentation sur un support choisi, délimité, qui donnera à la représentation valeur de symbole. Le choix de l'image, du pictural, est donc motivé par l'idée de mettre en lumière l'aspect de l'écrit relégué par les définitions du rapport épistémique au savoir, c'est-à-dire l'importance capitale d'éléments antéprédicatifs : la surface, l'écran (l'intention ?) du geste graphique, importance tout aussi capitale que celle du langage, dans la mesure où cet écran attribue aux figures le caractère de symboles. L'écran et ses contours sémantisent les traces qui y figurent tout autant que ce qu'elles représentent, à quoi elles réfèrent.

\section{La diversité des langues et des représentations comme point de départ}

Tout apprentissage peut être vu comme une modification des représentations, un changement, une mise en déséquilibre de ce que celui qui apprend savait ou croyait savoir. Pour que ce déséquilibre soit possible, il faut que le sujet apprenant soit assuré par un "ailleurs". "Pour accéder à cet espace de construction de connaissances ainsi perçu comme dangereux, menaçant, il convient de disposer d'un ailleurs compensatoire, d'un nid symbolique et imaginaire où se réfugier, d'un ancrage où notre parole est reconnue valeureuse et d'où elle assigne à autrui des places et des représentations familières." Pour que cet ailleurs compensatoire ne soit pas menacé à l'école, celle-ci devrait laisser une place à "ce qui le constitue intimement, la langue qu'on y parle et qui le soutient dans son advenir quotidien ${ }^{11}$.

L'école devrait donc, pour nêtre pas coupée de ses élèves, ne pas les amputer de leurs langues et de leur rapport à elles. Ne pas les ignorer, mais au contraire

8. Anne-Marie Christin, L'Image écrite ou la déraison graphique, Paris, Flammarion, 2009 [1995], p. 35. 9. Nancy Hornberger (dir.), Continua of Biliteracy. An Ecological Framework for Educationale Policy, Research, and Practice in Multilingual Settings. Clevedon, Multilingual Matters LTD, 2003. 10. Voir, notamment, François Dubet, L'École des chances. Qu'est-ce qu'une école juste?, Paris, Seuil, 2004. 11. Alain Coianiz, "Quand dire c'est se faire", in Paule Fioux (dir), Des langues de la maison aux langues de l'école en milieu plurilingue, Saint-Denis de la Réunion, Karthala, 2001, pp. 15-16. 
les accueillir et s'appuyer sur elles, qu'elles soient des variétés du français ou des langues plus éloignées. Il s'agit de construire un savoir commun, notamment langagier, qui se rapproche d'une norme scolaire implicitement attendue par l'institution, sans ignorer les pratiques et représentations des personnes qui composent principalement l'école, les élèves. À cette fin, Véronique Castellotti, partant du constat flagrant de la non-prise en compte des productions propres aux élèves, y compris "francophones", propose de "renverser la logique de l'éducation linguistique à l'école" en prenant appui sur la diversité linguistique et culturelle des élèves plutôt qu'en "s'appuyant sur une norme uniforme supposée a priori maîtrisée par tous ${ }^{12 "}$. Je tente d'adapter cette invitation à ma problématique en proposant de cesser de m'appuyer sur une conception de l'écrit uniforme a priori partagée par tous.

\section{Prendre en compte la pluralité des élèves}

Pour travailler avec des jeunes qui n'avaient pas été scolarisés avant leur arrivée en France, je me suis logiquement rendue dans une structure spécifiquement pensée pour eux, une classe d'accueil pour élèves nouvellement arrivés et peu ou pas scolarisés antérieurement (CLA-NSA ${ }^{13}$ ). Cette classe accueille des jeunes âgés de 11 à 16 ans qui ont en commun la migration, vécue seuls pour certains (mineurs isolés) ou en famille. Je suis également intervenue dans une classe de $3^{\text {e }}$ section d'enseignement général et professionnel adapté (Segpa), dont l'effectif de 16 élèves comprenait 7 élèves ayant migré. Parmi eux, 5 ont été scolarisés en CLA-NSA, 1 est arrivé sans scolarisation antérieure en $\mathrm{CE} 2$, et 1 a suivi une scolarisation ordinaire en Italie, pays dans lequel il est arrivé durant sa petite enfance. Les critères d'orientation en Segpa sont "des diffcultés scolaires graves et durables, auxquelles n'ont pu remédier les actions de prévention, d'aide et de soutien et l'allongement des cycles. Les élèves ne maîtrisent pas toutes les compétences et connaissances (...) attendues à la fin du cycle [2] et présentent (...) des lacunes importantes dans l'acquisition de celles prévues à l'issue du cycle [3]". Leur âge (d'une année ou plus supérieur à l'âge "normal" d'un élève de $3^{\mathrm{e}}$ ), ajouté à la courte durée de scolarisation de certains d'entre eux (près de $40 \%$ ), ne permet pourtant pas de penser qu'ils ont bénéficié de longues remédiations ou de maintien dans les cycles.

Les origines géographiques des élèves avec lesquels j’ai travaillé sont diverses : France, Kosovo, Maroc, Italie, Afghanistan, Maroc, Algérie, Angola, Arménie, Congo-Kinshasa, Mongolie, Sri Lanka, Tchad, Tchétchénie, Turquie.

Varient également leurs âges, II s'agit de construire les langues qu'ils connaissent, un savoir commun, leur niveau de français, leur notamment langagier, qui se niveau scolaire - qui est pour rapproche d'une norme scolaire tous considéré comme infé- implicitement attendue rieur au niveau normale- parl'institution, sans ignorer ment attendu à leur âge, ce les pratiques et représentations qui semble être le seul point des personnesqui composent commun que l'institution principalement l'école, leur reconnaît. La caractéris- les élèves. tique principale de ces classes, outre l'allophonie des élèves, tient aux yeux des enseignantes en deux mots : diversité, hétérogénéité. Mon intervention dans ces classes a également des origines diverses : le désir de mener une action auprès de jeunes migrants non scolarisés antérieurement, désir lié à un travail de thèse, et une demande institutionnelle de formation d'enseignant de Segpa accueillant des élèves non francophones et dits "en difficulté langagière ${ }^{14 "}$.

\section{La conception d'ateliers passerelles}

Partant de ces éléments, j’ai imaginé un dispositif dont je souhaitais qu'il soit à la fois ancré dans 
le contexte institutionnel et ouvert à l'expérience antérieure, en tout cas pas uniquement scolaire des élèves. Pour ce faire, je me suis appuyée sur les programmes d'arts plastiques au collège qui mettent en avant la sollicitation de l'initiative des élèves et la facilitation de l'accès à l'autonomie. "Cette part d'autonomie ainsi que les initiatives dont les élèves font preuve dans la conduite de leurs projets jusqu'à leur réalisation se mani-

S'ouvrir à l'expérience des

élèves, c'est aussi s'ouvrir

à leurs langues, et à la notion de compétence plurilingue et pluriculturelle festent aussi dans des situations d'échange oral, individuelles ou collectives. Du fait de la pratique, leur réflexion autour (...) de leur production est fondée sur un vécu qui nourrit l'expression orale. Cela peut également prendre la forme de notations écrites ${ }^{15}$." Je souhaitais également pouvoir répondre à la demande institutionnelle qui m'était soumise ${ }^{16}$. Mon intervention prendrait donc la forme d'ateliers d'arts plastiques qui permettraient, outre un détour pour atteindre des objectifs langagiers, de travailler à partir du concept de pensée-écran développé par Anne-Marie Christin, de mettre en lien les activités plastiques des élèves avec des pratiques d'écriture envisagées selon trois axes : la construction du récit, stimulant l'imaginaire ; le geste graphique, rapport sensible au signe et à son support ; et, de façon transversale aux deux précédents, la trace, l'inscription durable de la langue sur des supports variés.

Véronique Castellotti et Danièle Moore montrent que l'utilisation de dessins d'enfants, et des discours dont ils sont le support, permet de légitimer aux yeux de l'enfant le discours qu'il produit sur sa propre production, de "coconstruire un espace d'interprétation et ainsi d'ancrer la recherche dans l'action, notamment didactique ${ }^{17 "}$. L'approche des représentations par les arts plastiques devrait, en outre, ouvrir les possibilités de figuration des enfants au sensible et à l'imaginaire ${ }^{18}$, et non les restreindre au caractère épistémique de l'écrit scolaire. Les créations des élèves, mêlées à leurs discours, "construisent un poly-texte, qui demande de le comprendre pour, aussi, se comprendre ${ }^{19}$ ". J'interprète l'emploi du réfléchi "se" dans un sens mutuel, "se comprendre" signifierait alors "se comprendre l'un l'autre", et pour le chercheur, "comprendre l'élève". Le chercheur doit donc faire place à l'expérience du sujet avec lequel il travaille à la co-construction d'une interprétation. Or s'ouvrir à l'expérience des élèves, c'est aussi s'ouvrir à leurs langues, et la notion de compétence plurilingue et pluriculturelle permet de prêter attention à la "diversité des modalités de construction et d'évolution des ressources en lien avec les dimensions (...) cognitives, affectives et identitaires qui les accompagnent, [ainsi qu'aux] réglages identitaires auxquels se livre en permanence celui qui développe consciemment un profil plurilingue et pluriculturel ressenti comme un atout social ${ }^{20 "}$.

Ces dernières considérations théoriques et méthodologiques m’ont amenée à formuler comme suit les objectifs des ateliers : faire construire des représentations de l'écrit qui accordent une place aux expériences des élèves ; travailler la scripturalité de l'écrit ${ }^{21}$; proposer une approche qui laisse une place aux dimensions aussi bien sensibles et imaginaires que verbales de l'écriture ; développer une compétence plurilingue et pluriculturelle ; permettre aux élèves de réfléchir aux rôles et fonctions de l'écrit à l'école et hors de l'école.

\section{La mise en œuvre d'une expérience scripturale}

Lors des ateliers, j'ai soumis aux élèves les deux images suivantes qui ont servi de support à une réflexion collective sur le thème de l'écriture, soulevant notamment la question des liens et/ou des ruptures entre ce qui serait constitutif du dessin 


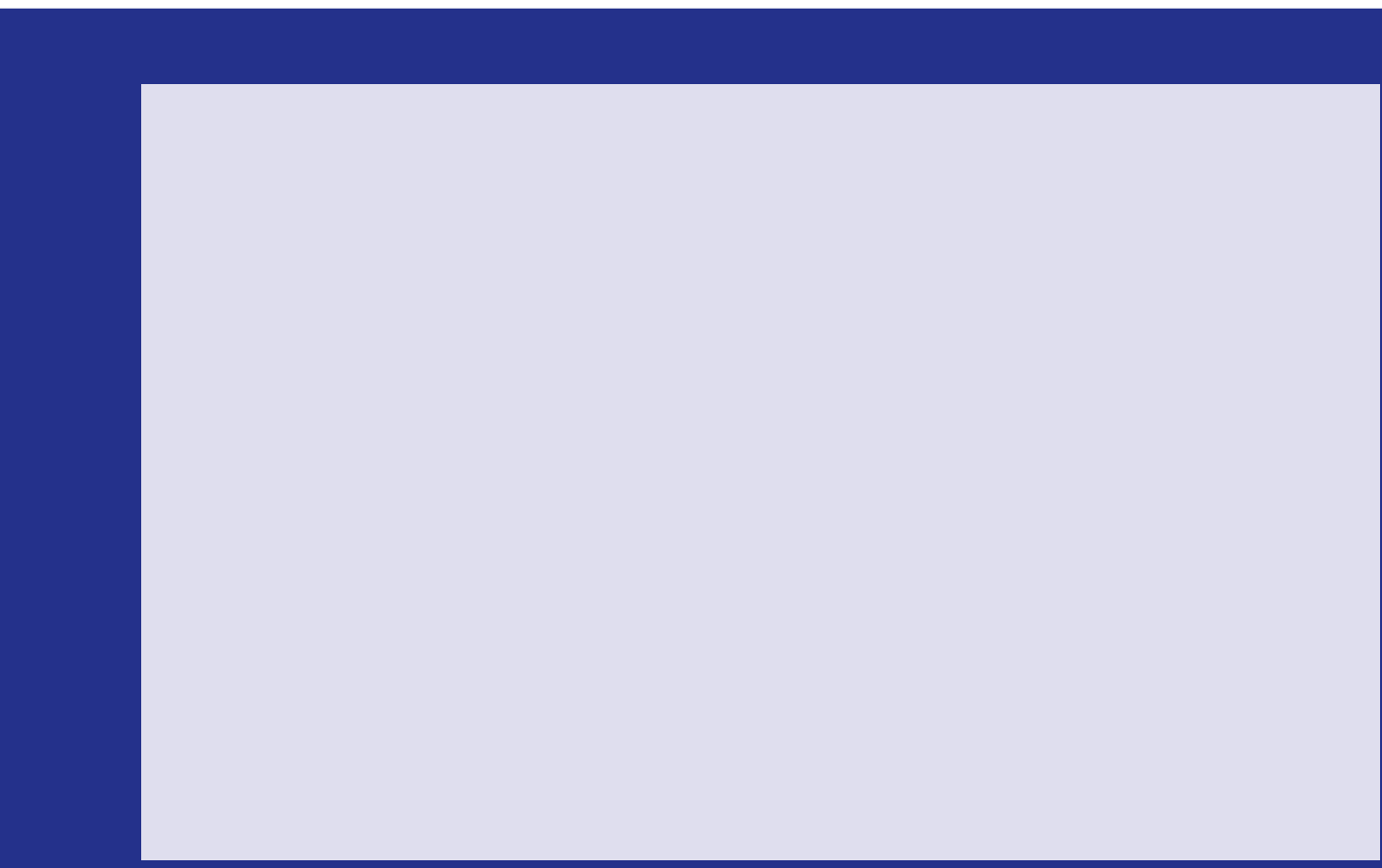

d'une part et de l'écriture d'autre part. Par la suite, les élèves ont été invités à produire un troisième élément, susceptible de poursuivre la série ainsi entamée ${ }^{22}$, puis des entretiens individuels ont fourni l'occasion aux auteurs des œuvres, les élèves, d'en livrer un commentaire. Ces entretiens ont également suscité des échanges autour des pratiques d'écriture des élèves interrogés.

Ces ateliers (répartis sur huit séances) et les discussions qui les ont accompagnés ont permis de mettre en lumière plusieurs éléments susceptibles de nourrir une réflexion sur la didactique de l'écrit dans le cas particulier de jeunes supposés faiblement littératiés ${ }^{23}$.

Tout d'abord, certains des élèves qui ont participé à cette recherche semblent avoir construit un rapport épistémique au savoir avant leur arrivée à lécole française : leurs pratiques montrent que, d'une part, ils utilisent l'écrit pour planifier des tâches, les structurer - mettant ainsi à distance l'objet qu'ils sont en train de créer -, et que, d'autre part, ils ont recours à des sources écrites, desquelles ils tirent un savoir, et qu'ils écrivent aussi pour apprendre (à écrire le français, notamment) et pour retenir leurs leçons.

Mais leurs pratiques d'écriture ne se limitent pas à ces aspects cognitifs. Elles endossent une grande diversité de fonctions : épistémiques, esthétiques. Les œuvres des élèves révèlent le goût et l'intérêt de certains d'entre eux pour les aspects purement scripturaux de l'écriture : la figure 3 "Fleur mongole", par exemple, dénote une attention particulière portée à la mise en espace de l'écrit et de ses ornementations, que l'on retrouve dans d'autres 
productions comme celles des figures 4 - avec le souci du cadre -, 5 et 6 .

Certains élèves nous ont aussi confié pratiquer l'écriture créative dans leur langue, poèmes en mongol, ou contes en arménien, par exemple. Pour d'autres, ces ateliers ont été l'occasion d'exprimer des appartenances et des compétences littératiées auxquelles l'école était jusque-là restée aveugle. Les figures 5 et 7 , très différentes l'une de l'autre, peuvent pourtant être rapprochées car elles introduisent à l'école des éléments de connaissance et de l'écriture d'autres langues (l'arabe pour la figure 7 et le gorane pour la figure 5).

Ces plurilittératies, qui apparaissent ici (figures 5 et 7) comme étant le fait d'élèves migrants qui ont construit des compétences hors de l'école, naissent aussi parfois au sein de la classe, à l'occasion précisément des ateliers proposés. C'est ce qu'illustre la figure 4, sur laquelle un élève de la CLA-NSA a pris l'initiative de collecter auprès de ses camarades des "échantillons" des différents systèmes d'écriture en présence. Cet exemple, mais aussi celui 
rencontré dans la classe de Segpa où un élève français "monolingue" a sollicité l'aide de certains de ses pairs pour écrire son prénom en caractères arabes, ajouté à l'observation des circulations qui se sont fait jour durant les ateliers, me semblent éclairer le plaisir et l'intérêt suscités par les expériences scolaires ou extrascolaires des pairs. Plaisir et intérêt qui opèrent comme source d'appropriation, de construction de connaissances propres à celui qui utilise pour son œuvre les expériences des autres.

Aux objectifs énoncés répondent les analyses - construites en collaboration avec les élèves - de leurs pratiques, de leurs œuvres commentées, de leurs représentations. Ces analyses tendent à montrer une grande diversité des pratiques et représentations, diversité qui, prise au sérieux, devrait constituer une ressource protéiforme pour la didactique de l'écrit. Ressource protéiforme, parce qu'elle revêt des atours linguistiques (Nathalie Auger a montré comment comparer les langues pour en apprendre d'autres), qu'elle permet une pédagogie du détour (par les arts plastiques notamment), qu'elle s'enracine dans l'expérience des élèves pour travailler leur créativité, mais encore parce qu'elle me semble permettre d'accompagner les élèves dans le tissage d'un réseau qui les aide à assumer leurs appartenances plurielles, évolutives et non exclusives les unes des autres.

\section{Conclusion}

C'est la mise en tension de deux conceptions de l'écrit qui a présidé à ce projet : d'un côté l'omniprésence à l'école d'un rapport épistémique au savoir fondé sur une mise en objet de la langue et du monde ${ }^{24}$, et de l'autre une conception métisse de la genèse de l'écrit insistant sur ses origines verbales (langagières) et graphiques (gestuelles, spatiales et sensibles). Du point de vue didactique, le choix a été fait de s'appuyer sur des approches centrées sur les élèves, leurs représentations, leurs productions, leur histoire. Il s'agissait de prendre appui sur la diversité constitutive des classes choisies pour la recherche pour construire, plus tard, une norme partagée. À cette norme devait initialement mener la prise de conscience d'éléments expérientiels

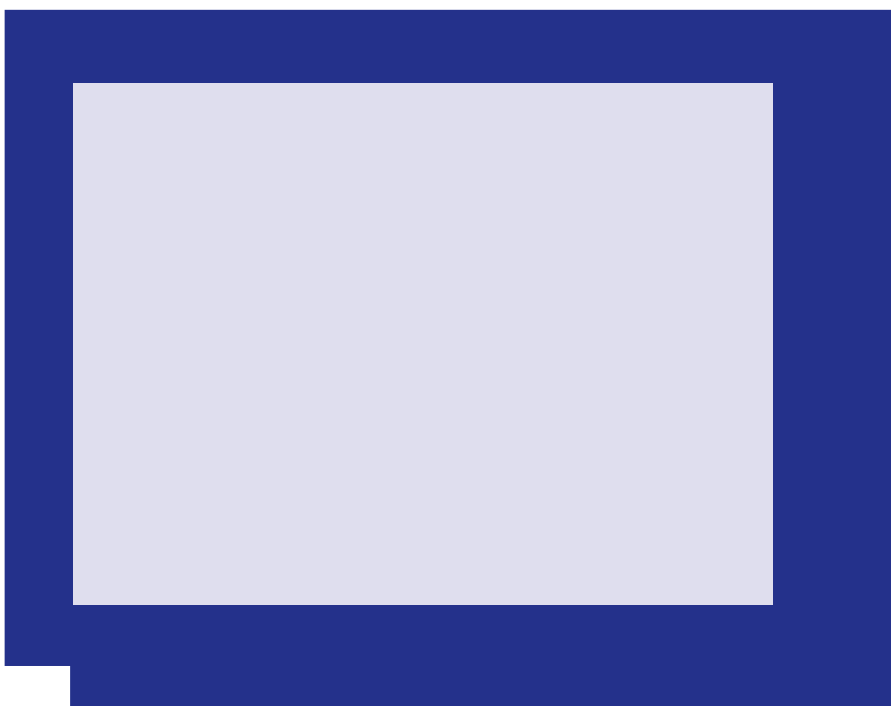

liés à la migration, qui, en déplaçant les élèves, aura aussi fait varier leur place sur un continuum plurilittératié ${ }^{5}$ tant du point de vue du contexte (devenu scolaire), des représentations et compétences en lien avec l'écrit, que des langues en jeu. Mais la mise en regard des objectifs initialement fixés et des analyses que j’ai pu livrer, largement appuyées sur les productions des élèves et leur engagement, me permettent de dépasser mes propres représentations. Cette recherche reposant sur la construction de sens par le chercheur et les autres sujets qui y sont engagés, dans un processus de co-interprétation, impose d'accepter l'inattendu, l'impensé, et même de compter sur eux. Suivant des orientations sous-jacentes, partant de ses questionnements, le chercheur, maître de ses 
analyses, se laisse déstabiliser par des rencontres singulières, posant ainsi la recherche comme une écriture de la pensée en migration, et la migration comme un processus d'altération (de modification) socialisée, c'est-à-dire qui se fait dans la rencontre. Des rencontres qui déstabilisent les représentations du chercheur, et le mettent en mouvement.

\section{Bibliographie}

Castellotti Véronique, "De la pluralité aux normes ou renverser la logique de l'éducation linguistique à l'école", in Martine Dreyfus, Jean-Marie Prieur, Hétérogénéité et variation. Perspectives sociolinguistiques, didactiques et anthropologiques, Paris, éd. M. Houdiart, 2012.

Castellotti Véronique, Moore Danièle, "Dessins d'enfants, recherche qualitative, interprétation. Des poly-textes pour l'étude des imaginaires du plurilinguisme", in Philippe Blanchet, Patrick Chardenet, Guide pour la recherche en didactique des langues, Paris, Archives contemporaines, 2011.

Castellotti Véronique, Moore Danièle, "La compétence plurilingue et pluriculturelle : genèse et évolutions d'une notion-concept", in Philippe Blanchet, Patrick Chardenet, Guide pour la recherche en didactique des langues, op. cit.

Charlot Bernard, Du rapport au savoir, Paris, Anthropos, 1997.

Christin Anne-Marie, L'Image écrite ou la déraison graphique, Paris, Flammarion, 1995.

Coianiz Alain, "Quand dire c'est se faire", in Paule Fioux (dir), Des langues de la maison aux langues de l'école en milieu plurilingue, Saint-Denis de la Réunion, Karthala, 2001.
Dubet François, L'École des chances. Qu'est-ce qu'une école juste?, Paris, Seuil, 2004.

Lahire Bernard, Culture écrite et inégalités scolaires. Sociologie de l'échec scolaire" à l'école primaire, Lyon, PUL, 2000.

Lorcerie Françoise, L'École et le Défi ethnique, Paris, INRP, 2003.

Lorilleux Joanna, "Regards croisés de familles et d'enseignants sur la scolarisation d'enfants roms allophones en Indre-et-Loire", mémoire de master II recherche, sous la direction de Véronique Castellotti, université François-Rabelais, Tours, 2011.

Lorilleux Joanna, "Le paradoxe de la classe, ou l'expérience formative à l'épreuve de la catégorisation”, in Glottopol, n² 23, 2014.

Poutignat Philippe, Streiff-Fenart Jocelyne, Théories de l'ethnicité, Paris, PUF, 1995.

Jacques Rancière, Le Maître ignorant. Cinq leçons sur l'émancipation intellectuelle, Paris, Fayard, 1987.

Williams Patrick, "L'écriture entre l'oral et l'écrit. Six scènes de la vie tzigane en France", in Daniel Fabre, Par écrit. Ethnologie des écritures quotidiennes, Paris, MSH, 1997. 


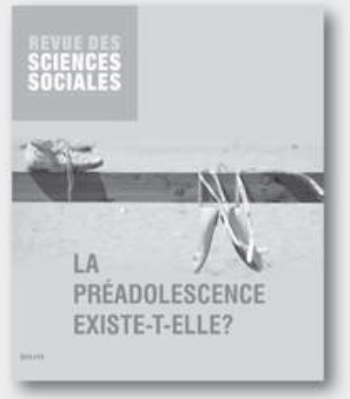

n512014 - prix 23,00 €

\section{LA PRÉADOLESCENCE EXISTE-T-ELLE?}

Depuis les années 1980 émerge une nouvelle catégorie d'âge, les préadolescents. Entre enfance dont ils n'auraient plus l'innocence et adolescence dont ils n'ont pas toutes les interrogations, de jeunes sujets se construisent dans un flou des dénominations et une indétermination des bornes d'âge.

La question des pubertés anticipées interroge le médical. Les concepteurs de produits nouveaux s'intéressent à ce marché. Les sociologues observent le développement d'une " sub-culture tween ". Les institutions cherchent à adapter leurs politiques socio-éducatives. L'abaissement de l'âge de la responsabilité légale pose des problèmes juridiques.

Alors que les frontières sociales et biologiques de l'enfance sont mises en question, une définition stable de la préadolescence s'affirme-t-elle pour autant?

\section{SOMMAIRE LA PRÉADOLESCENCE EXISTE-T-ELLE?}

\section{PRÉSENTATION}

NICOLETTA DIASIO \& VIRGINIE VINEL La préadolescence: un nouvel âge de la vie?

FLUIDITÉ DES TEMPS ET INSTABILITÉ DES CORPS

NICOLETTA DIASIO

Repenser la construction des âges: sortie de l'enfance et temporalités plurielles

SIMONA DE IULIO

Entre catégorisation et indétermination: l'imagerie des frontières de l'enfance dans la presse féminine et dans la presse « jeune $m$

DONATELLA COZZI

Corps en réseau. Jeunes et sexualité dans deux sites internet à Strabourg et Feltre (Vénétie)

MYRIAM KLINGER \& LOUIS MATHIOT

Reconfiguration relationnelle et travail du temps. Les rapports intergénérationnels lus par la presse des 9-15 ans

CRISTINA FIGUEIREDO

Enfance stable, adolescence instable chez les Touaregs du Gourma et de l'Adagh (Mali)

\section{CATÉGORISATIONS D'ÂGE}

ET ASSIGNATIONS SOCIALES

VIRGINIE VINEL

Controverses médicales autour de la puberté: précocité féminine et invisibilité masculine

LAURA DI SPURIO

"Làge de disgrâce ": les constructions de la prépuberté féminine en Belgique (1896-1960)

INGRID VOLÉRY

Gouverner la sortie de l'enfance par ses marges?

Les figures du caìd-de-cité et de la fille-de-quartier dans les espaces de l'animation socioculturelle
SIMONA TERSIGN

Grandir au prisme de l'ethnicisation en France et en Italie

CHRISTOPHE DARGĖRE

La triple peine: être préadolescents en institution médico-sociale

\section{SEUILS INSTITUTIONNELS}

ET TRANSITIONS ENFANTINES

p. 112 JULLE DELALANDE Devenir préadolescent et entrer au collège ANTONIETTA MIGLIORE Espaces de transition. Explorations de l'autonomie à la sortie de l'enfance

0. 126 JOËL ZAFFRAN

Entrer au collège, sortir de l'enfance. La part des loisirs culturels dans l'autonomisation des enfants

D. 134 MARIE-PIERRE JULLEN

Choisir ses vêtements et questionner l'incorporation des habitus

\section{CHANTIERS DE RECHERCHE}

DOSTENA ANGUELOVA-LAVERGNE

La fiction " société civile " et les think tanks bulgares. Ethnographie du discours anti-communiste des think tanks libéraux en Bulgarie (1989-2013)

GILBERT MEYNIEE

De l'hexagone au bled, un aristo, un anar: des écrivains français anticolonialistes?

LU - A LIRE

p. 174 Recensions

p. 180 Résumés des articles

Presses universitaires de Strasbourg - 5 allée du Gal Rouvillois - CS 50008- FR

67083 Strasbourg Cedex. Contact : pu-strasbourg@unistra.fr - Tél. 0368856265 - ISSN 1623-6572

www.revue-des-sciences-sociales.com 